\title{
Efeitos da terapia espelho na reabilitação do membro superior pós-acidente vascular cerebral
}

\author{
Luciana Protásio de Melo', Viviane Tavares Bezerra², Valton da Silva Costa ${ }^{3}$, \\ Felipe Hadan Mascarenhas de Souza², Júlio César Cunha da Silveira ${ }^{3}$
}

\begin{abstract}
RESUMO
O objetivo do estudo foi avaliar os efeitos da Terapia Espelho na recuperação funcional do membro superior de pacientes com sequelas crônicas de Acidente Vascular Cerebral. Neste estudo de caso, com abordagem qualitativa, a amostra foi composta por três indivíduos com idades de 51, 68 e 72 anos, submetidos a doze sessões de tratamento por quatro semanas. Foram avaliados antes e após o período de tratamento, utilizando-se a Escala Modificada de Ashworth, a Escala de Fugl-Meyer e a Medida de Independência Funcional. Como resultados foram observadas a diminuição da espasticidade em dois pacientes, e melhora modesta da função do membro superior parético além de independência funcional de todos os pacientes. Conclui-se que o uso da Terapia Espelho produziu alterações benéficas, principalmente quanto ao grau de espasticidade e função motora do membro superior parético. Ainda é necessária a realização de estudos com amostras mais amplas, para resultados mais consistentes e amplos.
\end{abstract}

Descritores: Acidente Vascular Cerebral; Paresia; Extremidade Superior; Reabilitação.

\section{Effects of mirror therapy in upper limb rehabilitation after stroke}

\begin{abstract}
The objective of the study was to evaluate the effects of mirror therapy on functional recovery of the upper limb of patients with chronic sequels of stroke. In this case study with qualitative approach the sample was composed of three individuals with ages 51, 68 and 72 years. They were submitted to twelve treatment sessions during four weeks, assessed before and after the treatment period using the Modified Ashworth Scale, the Fugl-Meyer Scale, and the Functional Independence Measure. As a result, a reduction of spasticity in two patients, and modest improvement in upper limb function besides functional independence of all patients were observed. It can be conclude that the use of Mirror Therapy produced beneficial alterations mainly in the degree of spasticity and motor function of the upper limb. It is still necessary carrying out studies with larger samples for more consistent results.
\end{abstract}

Descriptors: Stroke; Paresis; Upper Extremity; Rehabilitation.

\footnotetext{
${ }^{1}$ Mestre em Fisioterapia pela Faculdade Estácio do Rio Grande do Norte (FATERN), Natal, RN, Brasil.

${ }^{2}$ Fisioterapeuta pela Faculdade Estácio do Rio Grande do Norte (FATERN), Natal, RN, Brasil.

${ }^{3}$ Discente de Fisioterapia pela Faculdade Estácio do Rio Grande do Norte (FATERN), Natal, RN, Brasil.
} 


\section{Introdução}

O Acidente Vascular Cerebral (AVC) é uma das maiores causas de morte e sequelas neurológicas no mundo contemporâneo, com maior incidência em países não desenvolvidos ou em desenvolvimento ${ }^{1,2}$. No Brasil, em 2010, o AVC foi a segunda maior causa de mortalidade na população, responsável pela taxa de 52,3 mortes por 100.000 habitantes $^{3}$.

As sequelas após o AVC podem se modificar ao longo do tempo e implicar em graus variados de disfunção. A diminuição da independência em atividades funcionais da vida diária e a consequente dependência de terceiros configuram limitações importantes na vida desses pacientes ${ }^{4}$. Na fase crônica, o indivíduo pode apresentar hemiparesia com hipertonia espástica, hiperreflexia, déficits sensoriais e sinergias. 0 membro superior parético encontra-se limitado durante as atividades motoras, desde as mais simples até as mais complexas. Tais restrições surgem em virtude de um padrão hipertônico, diminuição de força e amplitude de movimento ${ }^{5,6}$.

A reabilitação precoce tem sido relacionada com recuperação funcional mais rápida e retardo no surgimento de sequelas motoras crônicas ${ }^{7}$. Várias técnicas de neuroreabilitação são utilizadas pela Fisioterapia, principalmente para reeducação neuromuscular do paciente após o AVC. Esses programas de reabilitação vêm contando com novas terapias, que estão sendo estudadas na atualidade e são promissoras, dentre elas a Terapia Espelho (TE).

Os primeiros estudos em relação à utilização da TE foram iniciados pelo neurologista indiano Ramachandran, na década de 90, em pacientes amputados que referiam a chamada dor fantasma, obtendo bons resultados ${ }^{8}$. Esta técnica foi proposta poucos anos depois para a reabilitação de pacientes com sequelas de AVC. A ideia da técnica é reeducar o cérebro com a execução de uma simples tarefa, onde o indivíduo realiza uma série de movimentos com o braço não afetado, sendo que este é visualizado em um espelho como se fosse o braço afetado. Dessa forma, pretende-se "enganar" o cérebro através da visualização do reflexo do braço não afetado no espelho, gerando estimulação sensorial por vias aferentes proprioceptivas e sinestésicas, onde a visualização de uma habilidade motora promove, então, a ativação na área visual cortical e em áreas envolvidas no comportamento motor. Pensa-se que a integração central dessa imagem estimulará fenômenos da neuroplasticidade, que podem constituir uma valiosa ferramenta na terapêutica aplicada aos pacientes pós-AVC ${ }^{9-13}$.

Os neurônios-espelho foram descobertos, na década de 90 , na área pré-motora de macacos ${ }^{14}$. Resultados de estudos de neuroimagem indicam que existe, nos seres humanos, um sistema de neurônios-espelho que estão distribuídos em várias áreas corticais frontoparietais análogas aos dos macacos, que são ativados durante a execução/observação de ações realizadas com a mão, com a boca e com os pés ${ }^{15,16}$. Acredita-se que o surgimento e o aprimoramento dessas células propiciam o desenvolvimento de funções importantes como linguagem, imitação e aprendizado. Dessa forma, a contribuição dos neurônios-espelho no processo de reabilitação de pacientes com sequelas de AVC pode ser muito importante ${ }^{17}$.

Diante do exposto, o objetivo deste estudo foi avaliar os efeitos da Terapia Espelho na recuperação funcional do membro superior de pacientes com sequelas crônicas de AVC.

\section{Metodologia}

Esta pesquisa caracteriza-se como um estudo observacional descritivo, de abordagem qualitativa, do tipo estudo de caso.

\section{Amostra}

Os participantes deste estudo foram recrutados do cadastro de pacientes da Clínica Escola da Faculdade Estácio do Rio Grande do Norte (FATERN), após o consentimento formal da instituição. A realização de todos os procedimentos foi esclarecida aos pacientes que, então, assinaram um Termo de Consentimento Livre e Esclarecido.

A amostra foi composta inicialmente por cinco pacientes com diagnóstico clínico de AVC. Foram establecidos como critérios de inclusão pacientes de ambos os sexos, com idade entre 45 e 75 anos, escolarizados/alfabetizados, com lesão unilateral e tempo de sequela mínimo de seis meses, ausência de déficit cognitivo avaliado pelo Mini-Exame do Estado Mental (MEEM), ausência de afasia severa, e ausência de espasticidade grave no membro superior parético (grau 4 na Escala Modificada de Ashworth). ${ }^{19} \mathrm{O}$ critério de exclusão adotado foi falta superior a $20 \%$ das sessões. Dois pacientes desistiram do estudo durante o processo, deixando de fazer parte da amostra. Portanto, a amostra final contou com três pacientes. Na Tabela 1 estão relacionadas as características gerais da amostra. 
Tabela 1 - Características gerais da amostra

\begin{tabular}{c|c|c|c|c}
\hline $\begin{array}{c}\text { Paciente/ldade/ } \\
\text { Gênero }\end{array}$ & $\begin{array}{c}\text { Tipo e Tempo de } \\
\text { AVC }\end{array}$ & Escore no MEEM & $\begin{array}{c}\text { Tratamento } \\
\text { paralelo }\end{array}$ & Presença de dor \\
\hline P1, 72, masculino & $\begin{array}{c}\text { Isquêmico, 42 } \\
\text { meses }\end{array}$ & 27 & Não & $\begin{array}{c}\text { Ombro parético, } \\
\text { movimentos ativos } \\
\text { acima de } 90^{\circ}\end{array}$ \\
\hline P2, 68, feminino & $\begin{array}{c}\text { Isquêmico, 7 } \\
\text { meses }\end{array}$ & 19 & Não & Joelhos e MSE \\
\hline P3, 51, masculino & $\begin{array}{c}\text { Hemorrágico, 7 } \\
\text { meses }\end{array}$ & 26 & Fisioterapia \\
convencional & $\begin{array}{c}\text { Ombro parético, } \\
\text { movimentos ativos } \\
\text { acima de } 90^{\circ}\end{array}$ \\
\hline
\end{tabular}

MEEM = Mini-Exame do Estado Mental (máximo 30 pontos).

$P=$ Paciente.

MSE $=$ Membro Superior Esquerdo.

\section{Instrumentos}

O MEEM foi utilizado para avaliar o estado cognitivo dos pacientes. Para avaliar a espasticidade do membro superior afetado foi usada a Escala Modificada de Ashworth. A função do membro superior comprometido do paciente foi avaliada pela Escala de Fugl-Meyer utilizando-se apenas o subitem do membro superior ${ }^{20}$ e a avaliação funcional do paciente foi feita pela Medida de Independência Funcional (MIF) ${ }^{21}$.

\section{Procedimentos}

A pesquisa foi submetida e aprovada pelo Comitê de Ética e Pesquisa da Universidade Federal do Rio Grande do Norte, sob o parecer número 163/2012 e número de protocolo 245/11, de 29 de fevereiro de 2012. Foram respeitados os aspectos éticos da Resolução $n^{\circ}$ 196/96 do Conselho Nacional de Saúde (CNS).

O estudo consistiu em uma avaliação inicial, com 12 sessões de intervenção e uma reavaliação após a conclusão do tratamento. Inicialmente, foram colhidos dados de identificação, anamnese e exame físico. Essa avaliação foi realizada por dois avaliadores com base na ficha de avaliação clínica em neurologia da instituição onde foi realizada a pesquisa, juntamente com os instrumentos supracitados. A reavaliação foi feita pelos mesmos avaliadores.

$\mathrm{Na}$ sessão seguinte, iniciou-se a intervenção com uso da TE. O protocolo adotado para a intervenção teve duração de quatro semanas, sendo três sessões semanais realizadas na Clínica de Fisioterapia Neurológica da FATERN. Seguindo o protocolo, foram realizados três séries de oito repetições nas três primeiras sessões de intervenção para cada movimento. Subsequentemente, foram realizadas três séries de dez repetições aplicadas entre a quarta e a sexta sessão. Da sétima à décima sessão, três séries de 12 repetições, e por fim, quatro séries de oito repetições aplicadas na décima primeira e décima segunda sessões de intervenção. O protocolo, baseado em estudos prévios, consistiu de movimentos com a mão não-parética, tendo seus movimentos refletidos através de um espelho. Antes do início e ao término de cada atendimento, os sinais vitais foram aferidos. Ao final de cada sessão, foi realizado alongamento passivo da cadeia muscular anterior do membro superior parético e mobilização intra-articular em cotovelo, punho e dedos. Cada sessão teve duração de 40 minutos.

Durante o tratamento, foi usada uma caixa de madeira com as seguintes dimensões: $58 \times 63 \times 26,5 \mathrm{~cm}$, com um espelho posicionado no meio, sendo cada quadrante de $29 \mathrm{~cm}$. Essa caixa foi posicionada no centro de uma mesa e o paciente ficou sentado em uma cadeira (quadril, joelhos e tornozelos a 90 graus) de frente para a mesa e a caixa. Os membros superiores do paciente foram posicionados em cada compartimento da caixa, sendo que a mão parética ficou no quadrante atrás do espelho e a não parética no quadrante à frente do espelho (Figura 1). Ao paciente, foram solicitados os seguintes movimentos do membro superior sadio: flexão de dedos, extensão de dedos, extensão de punho, movimento de pinça, pronação e supinação de antebraço. Após as 12 sessões de intervenção, o paciente foi submetido a uma reavaliação, utilizando-se os mesmos instrumentos iniciais. 
Figura 1 - Posicionamento do paciente durante TE (paciente visualiza o compartimento do MS sadio)

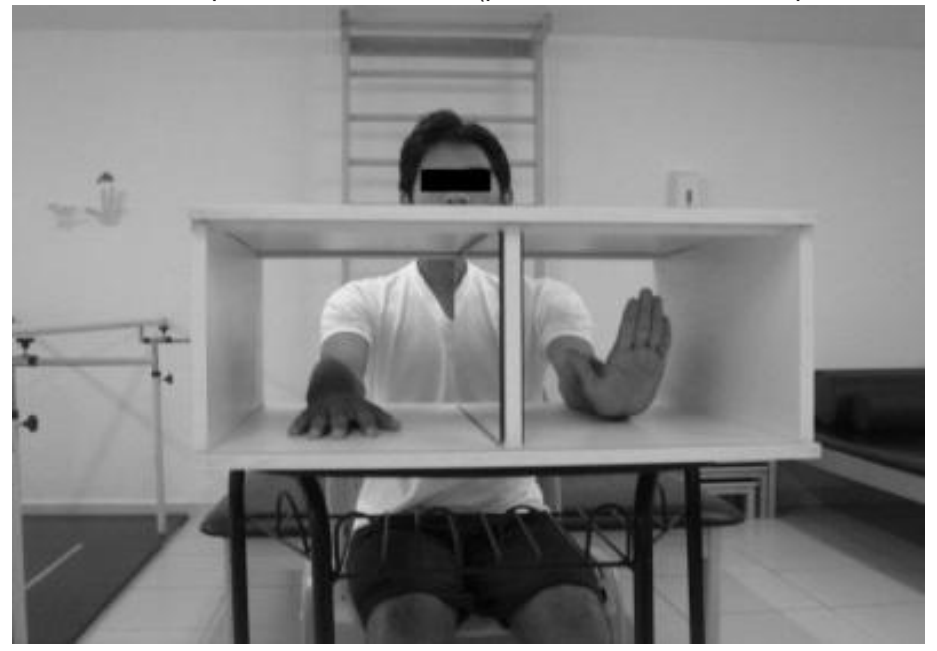

Análise dos dados

Os dados foram analisados de maneira descritiva, com comparações dos escores iniciais e finais dos instrumentos Escala Modificada de Ashworth, Escala de Fugl-Meyer e MIF.

\section{Resultados}

Os resultados do estudo podem ser visualizados na Tabela 2. 0 paciente 1 (P1) apresentava espasticidade leve nos flexores do cotovelo direito, que manteve-se após o uso da TE, porém, houve aumento da espasticidade dos flexores dos dedos; foram observadas ainda melhora da função motora para os movimentos do punho e mão, e do nível de independência funcional na MIF, principalmente nos subitens de vestir a metade superior e inferior do corpo e nas transferências, atingindo a independência total destes.

O paciente 2 (P2) apresentava espasticidade moderada nos flexores de cotovelo, punho, mão e dedos esquerdos. Esse grau de espasticidade obteve melhora em todos os grupos musculares. Quanto à função motora e independência funcional, não foram observadas melhoras significativas.

O paciente 3 (P3) apresentava espasticidade moderada em flexores de cotovelo, punho, mão e dedos esquerdos. Observou-se diminuição da espasticidade dos flexores de dedos e punhos, embora o mesmo não tenha ocorrido no mesmo grau nos músculos do cotovelo e ombro. Houve melhora modesta da função motora. Na avaliação da independência funcional, este paciente obteve aumento nos escores da MIF, atingindo independência completa nos subitens de vestir metade superior do corpo e utilização do vaso sanitário.

Tabela 2 - Resultados após quatro semanas de intervenção

\begin{tabular}{c|c|c|c}
\hline & & Antes & Depois \\
\hline \multirow{2}{*}{$\begin{array}{c}\text { Escala Modificada } \\
\text { de Ashworth (dedos, } \\
\text { punho, cotovelo, ombro } \\
\text { respectivamente) }\end{array}$} & $\mathrm{P} 1$ & $0,0,1,0$ & $1,0,1,0$ \\
\cline { 2 - 4 } & $\mathrm{P} 2$ & $3,3,2,2$ & $1+, 1+, 1+, 1+$ \\
\hline \multirow{2}{*}{\begin{tabular}{c} 
Escala de Fugl - Meyer \\
\cline { 2 - 4 }
\end{tabular}} & $\mathrm{P} 3$ & $1+, 2,2,1+$ & $1,1,2,1+$ \\
\cline { 2 - 4 } & $\mathrm{P} 1$ & Escore 58 & 67 \\
\hline \multirow{2}{*}{$\begin{array}{c}\text { Medida de Independência } \\
\text { Funcional }\end{array}$} & $\mathrm{P} 2$ & Escore 15 & 16 \\
\cline { 2 - 4 } & $\mathrm{P} 3$ & Escore 18 & 87 \\
\cline { 2 - 4 } & $\mathrm{P} 1$ & 62 & 63 \\
\hline
\end{tabular}

$P=$ Paciente.

Nota: Escala Modificada de Ashworth (espasticidade): 0 = ausente; $1,1+=$ leve; 3 = moderada; 4 = grave. 


\section{Discussão}

O presente estudo teve como foco uma nova terapia (TE) na recuperação funcional de pacientes de AVC e foi, nele, observada melhora dos movimentos de punho e dedos do P1, embora não se possa afirmar que essa melhora tenha se dado exclusivamente pela utilização da TE, ou se em conjunto com a mobilização intra-articular e alongamentos passivos, que foram realizados ao final de cada sessão. O P2 não apresentou melhora significativa. Este desfecho pode ter ocorrido devido ao fato de que ele apresentava uma comorbidade reumática (artrose), resultando em dores durante as sessões e limitando a aplicação do protocolo. 0 paciente 3 apresentou diminuição da espasticidade nos músculos flexores dos dedos e punho esquerdos, sem modificações evidentes nos músculos do cotovelo e ombro esquerdos.

A aplicação da TE promoveu uma tendência na diminuição da espasticidade do membro superior parético dos pacientes, quando não associada à dor, fato que não foi observado em outras amostras ${ }^{22}$, embora fosse observada melhora da função motora. $O$ estudo de cinco semanas corrobora os achados supracitados dessa pesquisa, havendo melhora motora embora não o suficiente para tornar a extremidade ativa ${ }^{23}$.

Uma pesquisa do ano de 2003 revelou resultados semelhantes com os desse estudo, ao observar melhora dos escores da escala de Fugl-Meyer no arco de movimento e velocidade da mão ${ }^{24}$. Ressalta-se que todos os estudos citados anteriormente foram realizados com pacientes nas fases aguda e subaguda, enquanto que, nesta pesquisa, todos os três indivíduos encontravam-se em fase crônica. Esse fato talvez possa explicar a diferença de resultados em relação a estudos prévios, visto que a cronicidade das sequelas pode gerar diferentes padrões de recuperação.

Em 2005, foi comprovado que a utilização do estímulo visual associado à prática física pode modificar o desempenho de uma tarefa motora, sendo que tais modificações estão associadas a mudanças fisiológicas e plásticas em nível cerebral $^{13}$. O mesmo também foi observado em um estudo de $2007^{25}$ relatando os mecanismos que parecem explicar a eficiência da TE na recuperação funcional do membro parético, ainda não esclarecidos completamente, mas que parecem estar ligados à neuroplasticidade. É consenso na literatura científica que a neuroplasticidade ocorre por meio do aprendizado de determinadas atividades ou pela repetição das mesmas, gerando adaptações plásticas e dinâmicas do sistema nervoso central, como a neurogênese e a sinaptogênese, ou modulações pré e pós-sinápticas, contribuindo para resultados positivos em resposta à reabilitação26-28.

Um estudo de caso, de um paciente com hemiparesia leve em fase crônica, revelou melhora de aspectos, como força de preensão palmar e amplitude de movimento do ombro, velocidade de execução dos movimentos nas tarefas motoras, bem como na distância de alcance funcional ${ }^{29}$. 0 protocolo, diferentemente deste estudo (que tem como base protocolos de vários estudos anteriores), teve duração de três meses, com prática domiciliar diária. Um estudo de caso já citado ${ }^{24}$, com 2 pacientes em fase crônica de AVC e hemiparesia leve, e duração semelhante ao deste estudo, porém com tempo de intervenção de apenas 20 minutos, obteve resultados similares ao anteriormente citado ${ }^{29}$, fato que foi novamente observado em um terceiro estudo de caso com um paciente com hemiparesia leve na fase crônica de AVC após três semanas de intervenção utilizando o mesmo protocolo ${ }^{30}$. Em ambos os estudos, a TE foi associada à prática mental dos movimentos, utilizando-se imagens de computador. Fato esse que impede que a melhora dos pacientes seja atribuída somente à TE, o mesmo ocorreu no presente estudo, onde a TE foi associada a alongamentos e mobilização intra-articular, ao final das sessões.

O P3, diferentemente dois outros dois pacientes, recebeu tratamento convencional de fisioterapia enquanto participava deste estudo, porém essa foi direcionada ao membro inferior, equilíbrio e marcha.

Os estudos supracitados incluíram em seus protocolos a realização de movimentos bilaterais, enquanto que, neste estudo, foi adotada a realização de movimentos apenas do membro superior não afetado enquanto o outro permanecia em repouso. Foi observado que, durante a intervenção, ocorriam ocasionalmente movimentos reflexos da mão parética, devido à ilusão de movimento normal contralateral produzida pelo reflexo no espelho. Tais movimentos podem representar um efeito positivo no ganho de mobilidade ativa da mão. Este fato pode ser explicado pelo estímulo visual proporcionado pela TE, substituindo a propriocepção, a qual, com frequência, se encontra diminuída ou, até mesmo, ausente ${ }^{31}$. Estudos controlados randomizados com desenhos mais homogêneos e parâmetros de avaliação mais padronizados tem sido realizados nos últimos anos e têm demonstrado os benefícios da TE na recuperação motora e funcional tanto a curto quanto a longo prazo (3 a 6 meses) $)^{22,32-35}$.

O protocolo utilizado, na presente pesquisa, para os movimentos de dedos e punho, o número e a duração das sessões foram os mais frequentemente adotados por estudos anteriores sobre a TE até 0 ano de 2002, conforme mostrou uma revisão da literatura ${ }^{36}$. 0 tamanho reduzido da amostra, bem como o fato da TE não ter sido usada isolada, fizeram com que este estudo se diferenciasse dos demais. Assim, estes fatores precisam ser levados em consideração em futuros estudos. 


\section{Considerações Finais}

O uso da TE produziu alterações quanto ao grau de espasticidade, melhorando os movimentos passivos das articulações acometidas. 0 mesmo não se pôde verificar nos movimentos ativos. Por se tratar de uma terapia que envolve aprendizagem motora, o tempo destinado à intervenção foi razoável quando comparado a pesquisas anteriores, porém a amostra foi muito reduzida. É necessário, portanto, que pesquisas futuras utilizem amostras mais amplas, de modo que possam obter resultados mais significativos e amplos, utilizando também um tempo de intervenção maior, permitindo que o mecanismo de neuroplasticidade ocorra com maior propriedade, ao mesmo tempo em que avaliem variáveis, como atividade motora e capacidade funcional.

\section{Referências Bibliográficas}

1. Feigin VL, Lawes CMM, Bennett DA, Barker-Collo SL, Parag V. Worldwide stroke incidence and early case fatality reported in 56 population-based studies: a systematic review. Lancet Neurol. 2009;8(4):355-69.

2. Organización Mundial de la Salud. Estadísticas sanitarias mundiales [Internet]. 2012. Disponível em: http://www.who. int/gho/publications/world_health_statistics/ES_WHS2012_Full.pdf

3. Organização Pan-americana da Saúde. Saúde nas Américas: panorama regional e perfis de países [Internet]. Washington, DC: OPS, 2012. Disponível em: http://www1.paho.org/saludenlasamericas/docs/sa-2012-resumo.pdf

4. Polese JC, Tonial A, Jung FK, Mazuco R, Oliveira SG, Schuster RC. Avaliação da funcionalidade de indivíduos acometidos por Acidente Vascular Encefálico. Rev Neurocienc. 2008;16(3):175-78.

5. Sommerfeld DK, Eek EU, Svensson A, Holmqvist LW, Von Arbin MH. Spasticity after stroke: its occurrence and association with motor impairments and activity limitations. Stroke. 2004;35(1):134-40.

6. O'Sullivan S, Schmitz T. Fisioterapia: Avaliação e Tratamento. São Paulo: Manole; 2010.

7. Bai Y, Hu Y, Wu Y, Zhu Y, He Q, Jiang C, Sun L, Fan W. A prospective, randomized, single-blinded trial on the effect of early rehabilitation on daily activities and motor function of patients with hemorrhagic stroke. J. Clin. Neurosci. 2012;19(10):1376-79.

8. Ramachandran VS. Behavioral and magnetoencephalographic correlates of plasticity in the adult human brain. Proc Natl Acad Sci USA. 1993;90(22):10413-20.

9. Shepherd R, Carr J. Ciência do Movimento: Fundamentos para a Fisioterapia na Reabilitação. São Paulo: Manole; 2003. 10. Karmarkar A, Lieberman I. Mirror box therapy for complex regional pain syndrome. Anaesthesia. 2006;61(4):412-3.

11. Ramachandran VS, Rogers-Ramachandran D. Synaesthesia in phantom limbs induced with mirrors. Proc Biol Sci. 1996;263(1369):377-86.

12. Vladimir TYI, Geertzen JHB, Keizer D, Paul WC. Mirror box therapy added to cognitive behavioural therapy in three chronic complex regional pain syndrome type I patients: a pilot study. Int J Rehabil Res. 2007;30(2):181-8.

13. Ramachandran VS. Plasticity and functional recovery in neurology. Clin Med. 2005;5(4):368-73.

14. Rizzolatti G, Fadiga L, Gallese V, Fogassi L. Premotor cortex and the recognition of motor actions. Cogn Brain Res. 1996;3(2):131-41.

15. Buccino G, Binkofski F, Riggio L. The mirror neuron system and action recognition. Brain Lang. 2004;89(2):370-6.

16. Rizzolatti G, Arbib MA. Language within our grasp. Trends Neurosci. 1998;21(5):188-94.

17. Lameira AP, Gawryszewski LG, Pereira A. Neurônios espelho. Psicol USP. 2006;17(4):123-33.

18. Brucki SMD, Nitrini R, Caramelli P, Bertolucci PHF, Okamoto IH. Sugestões para o uso do Mini-Exame do estado mental no Brasil. Arq Neuro-Psiquiatr. 2003;61(3B):777-81.

19. Bohannon RW, Smith MB. Interrater reliability of a modified Ashworth scale of muscle spasticity. Phys Ther. 1987;67(2):206-7.

20. Maki T, Quagliato EMAB, Cacho EWA, Paz LPS, Nascimento NH, Inoque MMEA, et al. Estudo de confiabilidade da aplicação da Escala de Fugl-Meyer no Brasil. Rev Bras Fisioter. 2006;10(2):177-83.

21. Riberto M, Miyazaki MH, Jucá SSH, Sakamoto H, Pinto PPN, Battistella LR. Validação da versão brasileira da Medida de Independência Funcional. Acta Fisiátrica. 2004;11(2):72-6.

22. Yavuzer G, Selles R, Sezer N, Sütbeyaz S, Bussmann JB, Köseoğlu F, et al. Mirror therapy improves hand function in subacute stroke: a randomized controlled trial. Arch Phys Med Rehabil. 2008;89(3):393-8. 
23. Pereira LS, Gil LM, Souza WC. Técnica de mirror visual feedback em paciente hemiparético no pós-acidente vascular encefálico. Revista Eletrônica Novo Enfoque. 2010;10(10):113-8.

24. Stevens JA, Stoykov MEP. Using motor imagery in the rehabilitation of hemiparesis. Arch Phys Med Rehabil. 2003;84(7):1090-2. 25. Trevisan CM, Trevisan ME, Oliveira RM, Mota CB, Pratesi R. Reabilitação da hemiparesia pós-acidente vascular encefálico com mirror visual feedback. Fisioter Bras. 2007;8(6):452-4.

26. Johansson BB. Brain plasticity in health and disease. Keio J Med. 2004;53(4):231-46.

27. Nudo RJ, Plautz EJ, Frost SB. Role of adaptive plasticity in recovery of function after damage to motor cortex. Muscle Nerve. 2001;24(8):1000-19.

28. O'Connell MJ. Prediction of return to work following traumatic brain injury: Intellectual, memory, and demographic variables. Rehabil Psychol. 2000;45(2):212-7.

29. Sathian K, Greenspan Al, Wolf SL. Doing it with mirrors: a case study of a novel approach to neurorehabilitation. Neurorehabil Neural Repair. 2000;14(1):73-6.

30. Stevens JA, Stoykov MEP. Simulation of bilateral movement training through mirror reflection: a case report demonstrating an occupational therapy technique for hemiparesis. Top Stroke Rehabil. 2004;11(1):59-66.

31. Altschuler EL, Wisdom SB, Stone L, Foster C, Galasko D, Llewellyn DME, et al. Rehabilitation of hemiparesis after stroke with a mirror. Lancet. 1999;353(9169):2035-6.

32. Cacchio A, De Blasis E, Necozione S, Orio F, Santilli V. Mirror Therapy for Chronic Complex Regional Pain Syndrome Type 1 and Stroke. N Engl J Med. 2009a;361(6):634-6.

33. Cacchio A, De Blasis E, De Blasis V, Santilli V, Spacca G. Mirror therapy in complex regional pain syndrome type 1 of the upper limb in stroke patients. Neurorehabil Neural Repair. 2009b;23(8):792-9.

34. Dohle C, Pullen J, Nakaten A, Kust J, Rietz C, Karbe H. Mirror therapy promotes recovery from severe hemiparesis: a randomized controlled trial. Neurorehabil Neural Repair. 2009;23(3):209-17.

35. Michielsen ME, Smits M, Ribbers GM, Stam HJ, Geest JN, Bussmann JBJ, etal. The neuronal correlates of mirror therapy: an fMRI study on mirror induced visual illusions in patients with stroke. J Neurol Neurosurg Psychiatry. 2011;82(4):393-8.

36. Toh SFM, Fong KNK. Systematic review on the effectiveness of mirror therapy in training upper limb hemiparesis after stroke. Hong Kong J Occup Ther. 2002;22(2):84-95.

\section{Luciana Protásio de Melo}

Endereço para correspondência - Rua: Estrela do Mar, n²22, Bloco Brasil, apto 304, CEP: 59.151-120 Bairro: Nova Parnamirim, Cidade: Parnamirim, RN, Brasil.

E-mail: protasio.melo@yahoo.com.br

Lattes: http://lattes.cnpq.br/5823735725272248

Viviane Tavares Bezerra - vivianezinha23@hotmail.com

Valton da Silva Costa - valtontj@hotmail.com

Felipe Hadan Mascarenhas de Souza - felipe_hadan@hotmail.com

Júlio César Cunha da Silveira - julioccsilveira@hotmail.com

\section{Enviado em 26 de junho de 2014.} Aceito em 11 de dezembro de 2014. 
